\title{
Proposals for enhancements of topographic maps for high alpine tours
}

\author{
Stephan Wondrak ${ }^{\mathrm{a}}$ \\ ${ }^{a}$ Swiss Federal Statistical Office, stephan@wondrak.ch
}

Keywords: Topographic map, Map signature, Tour guide, Online portal, High alpine tours

\begin{abstract}
:
Today we have diverse and high-quality bases for the planning of high alpine tours. Most widely used are topographic maps, tour guides and online portals. In combination with a topographic map or a map extract, photos and topos ${ }^{1}$, the textual description of a tour guide gives us a comprehensive and detailed picture of our planned high alpine tour. However, this picture can still be improved. The character of a high alpine tour is determined by many criteria. Some of the most important ones can be examined with a topographic map:
\end{abstract}

- Length of the tour (distances, total time)

- Altitude profile (in relation to sea level, differences in altitude)

- Steepness and exposure of the terrain (steep faces, ridges)

- Land cover (rock, glacier, moraine, meadows, forest)

Other important criteria of a high alpine tour are described textually in tour guides and tour reports on the Internet ${ }^{2}$. Some of these criteria or properties remain unchanged over a long time, others can change within hours. Unchanging properties are for instance the

- Difficulties in climbing (according to international UIAA ${ }^{3}$ scale)

- Stability or fragility of the rocks

- Clarity of the terrain (structure, important for pathfinding)

Slowly changing properties are the

- Condition of glaciers (snow cover, crevasses, retreat)

- Stability of slopes (glacial moraines)

- Degree of protection (security hooks, safety rods, fixed ropes, rappelling points)

- Marking of the route (Steinmänner, coloured markings)

- Number of rope teams on the route (potential waiting times, traffic jams, oncoming traffic)

Suddenly changing properties are the

- Weather conditions (temperature, wind, clouds, rain- or snowfall, sight, insolation)

- Conditions on the terrain (fresh snow, moisture, icing of the rocks)

- Rock or ice fall

From the cartographic perspective, in particular the unchanging or slowly changing properties are of interest. How can these properties be mapped in an accurate and comprehensible way?

This presentation focuses on potential enhancements of topographic maps, topos and tour guides, to map the abovenamed unchanging or slowly changing properties of high alpine tours. In this context, the avoidance of overcrowding of the already dense topographic maps is an important requirement, especially for the printed versions of these maps. In interactive online maps, the new map content can be added with a new map layer. Tour guides often include extracts of a topographic map, where requirements and constraints for new map content may be not as strong as in the original printed version. The potential for enhancements of the existing planning bases for high alpine tours is considerable. Due to global warming, route conditions are changing quickly. Unexpected situations on high alpine tours emphasize the necessity of an enhancement or supplement of the existing cartographic representations.

\footnotetext{
${ }^{1}$ The graphical representation of a climbing route with sketch drawing

${ }^{2}$ These descriptions are often supplemented by photos

${ }^{3}$ International Climbing and Mountaineering Federation
} 